\title{
Testing of Lagrange multiplier damped least-squares control algorithm for woofer-tweeter adaptive optics
}

\author{
Weiyao Zou* and Stephen A. Burns \\ School of Optometry, Indiana University, 800 East Atwater Avenue, Bloomington, Indiana 47405, USA \\ ${ }^{*}$ Corresponding author: zouweiyao @ gmail.com
}

Received 8 November 2011; revised 12 December 2011; accepted 13 December 2011; posted 13 December 2011 (Doc. ID 157803); published 12 March 2012

\begin{abstract}
A Lagrange multiplier-based damped least-squares control algorithm for woofer-tweeter (W-T) dual deformable-mirror (DM) adaptive optics (AO) is tested with a breadboard system. We show that the algorithm can complementarily command the two DMs to correct wavefront aberrations within a single optimization process: the woofer DM correcting the high-stroke, low-order aberrations, and the tweeter DM correcting the low-stroke, high-order aberrations. The optimal damping factor for a DM is found to be the median of the eigenvalue spectrum of the influence matrix of that DM. Wavefront control accuracy is maximized with the optimized control parameters. For the breadboard system, the residual wavefront error can be controlled to the precision of $0.03 \mu \mathrm{m}$ in root mean square. The W-T dual-DM AO has applications in both ophthalmology and astronomy. (C) 2012 Optical Society of America

OCIS codes: $\quad 010.1080,220.1080,170.1790,330.4460$.
\end{abstract}

\section{Introduction}

The dynamic nature of human eye optics requires that an adaptive optics (AO) retinal imaging system corrects both low- and high-order wavefront aberrations simultaneously. While trial lenses can be used to correct defocus and astigmatism, this approach is limited and cumbersome due to the fact that not all of the low-order aberrations can be well compensated by trial lenses. Furthermore, it requires an extra step in subject testing and it may require changing the combination and orientation of the trial lenses due to the variation in refractive error across the retina [1-6]. In addition, even if the static low-order aberrations are corrected, unless powerful cycloplegic drugs are used, defocus can vary dynamically during in vivo retinal imaging due to eye accommodations [7]. A single deformable-mirror (DM) can make excellent wavefront corrections if it has relatively high actuator counts and ample stroke. However, most precision high-order mirrors, such as the microelectromechanical systems (MEMS) mirrors, have actua-

$1559-128 \mathrm{X} / 12 / 091198-11 \$ 15.00 / 0$

(ㄷ) 2012 Optical Society of America tor stroke limitations. For this reason, as well as for cost considerations, the woofer-tweeter (W-T) approach is very attractive [8-10].

The W-T AO is a special configuration of dual-DM AO. The AO system with two DMs was first reported for compensating amplitude and phase in wavefront propagation through turbulent medium [11-13]. In ocular AO imaging, the W-T approach was first implemented with a sequential dual-step correction [8-10], where a woofer DM is used for correcting the low-order aberrations and a tweeter DM for highorder aberrations. First, the woofer was optimized for wavefront correction to convergence, and then the AO control was switched to the tweeter optimization. Because of the rapid variations of the wavefront aberrations of the human eye [14,15], the sequential approach can be cumbersome and require alternating steps. Simultaneous correction algorithms for W-T AO systems have been implemented [16,-19]. $\mathrm{Hu}$ et al. proposed to split the Zernike coefficients reconstructed from slope data and estimate the DM commands independently from the split Zernike coefficients [20]. Lavigne and Véran proposed implementing the $\overline{\mathrm{W}}$-T split in Fourier space, with the lowspatial-order Fourier modes off-loaded to the woofer 
DM and the higher-spatial-order modes to the tweeter DM, and then the DM commands were obtained with a Fourier reconstructor [21]. Conan et al. proposed concatenating the influence matrices of the tip/tilt DM, the woofer DM, and the tweeter DM into a single matrix for zonal control, and the actuator commands of the three DMs were obtained by evaluating the pseudo inverse of the concatenated matrix [22]. For the modal control algorithm, the influence matrix used would be a new set of influence functions built from a given set (such as the tweeter) such that it is orthogonal to the third set (i.e., the woofer) [22]. Recently Li et al. proposed a zonal algorithm to construct a new influence matrix for the tweeter that is orthogonal to the woofer influence matrix, so the woofer DM and tweeter DM work independently in wavefront correction for ocular retinal imaging [23]. In 2008 , we proposed to construct a composite influence matrix from the influence matrices of the two DMs with the Lagrange multiplier (LM) method, so the commands of the two DMs could be directly computed from wavefront measurements. To suppress the correlation between the two DMs, the damped least squares (DLS) method was employed [24,25].

The LM DLS method can provide simultaneous AO corrections for ocular imaging and astronomical imaging. In vivo retinal imaging using the LM DLS method was reported recently [26]. In this paper, we present the testing of the LM DLS control algorithm on a breadboard W-T AO system. Section $\underline{2}$ describes the experimental setup and the related methodology for implementation. Section 3 describes the experimental results of wavefront correction, and we demonstrate an approach to optimizing the control parameters. In Section 4 , we discuss the optimal control parameters and compare the wavefront fitting errors between the 1-Step and 2-Step inverse computations. Section $\underline{5}$ is the conclusion.

\section{Methods}

We first briefly summarize the experimental setup of the breadboard W-T AO system, and then describe the correlation (coupling) suppression in the dualDM AO control. We validate the proposed algorithm by inspecting the wavefront partition and correction of the control through simulations.

\section{A. Experimental Setup}

The experimental system for the W-T dual-DM AO is shown in Fig. 1. A 52-actuator electromagnetic DM produced by Imagine Eyes (Mirao-52d DM, maximum stroke $50 \mu \mathrm{m}$ ) was used as the woofer [27], and a 140-actuator DM produced by Boston Micromachines Corp. (BMC DM, maximum stroke $2.5 \mu \mathrm{m}$ ) was used as the tweeter [28]. The light source was a superluminescent laser diode (SLD, Superlum) with a central wavelength of $676.6 \mathrm{~nm}$ and a full-width at half-maximum spectral bandwidth of $13.9 \mathrm{~nm}$ [29]. Light from the SLD was collimated and passed though an aberration sample, which was a piece of poured glass or a trial lens. The aberrated wavefront

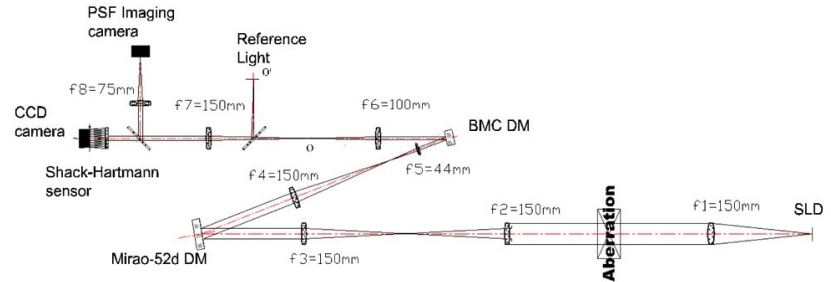

Fig. 1. (Color online) Optical layout of the WT AO breadboard system.

was corrected by both the Mirao-52d DM (15 mm in diameter) and the BMC DM $(4.4 \mathrm{~mm} \times 4.4 \mathrm{~mm}) \mathrm{si}$ multaneously. A Shack-Hartmann (SH) wavefront sensor was placed in downstream of the correcting mirrors for wavefront measurement. The aberration sample, the Mirao-52d DM, the BMC DM, and the lenslet array of the $\mathrm{SH}$ wavefront sensor were optically conjugated to one another by the relay lenses. The pupil sizes at the aberration sample, the Mirao$52 \mathrm{~d} \mathrm{DM}$, and the BMC DM were $\Phi 15 \mathrm{~mm}, \Phi 15 \mathrm{~mm}$, and $\Phi 4.4 \mathrm{~mm}$, respectively. The SH wavefront sensor consisted of a lenslet array and a charge-coupled device $(\mathrm{CCD})$ camera $[30,31]$. The pupil size at the location of the lenslet array was $\Phi 6 \mathrm{~mm}$, using a $19 \times 19$ array of lenslets. The pitch size of the lenslet was $0.3 \mathrm{~mm}$ and the focal length $7.6 \mathrm{~mm}$. The reference $\mathrm{SH}$ grid was generated by imaging collimated light from a $\Phi 200 \mu \mathrm{m}$ pinhole illuminated by a lightemitting diode (LED). A separate CCD camera was used to measure the imaging system's point spread function (PSF) [31]. Figure 2 shows the actuator distributions of the Mirao DM and the BMC DM. Both mirrors are optically conjugate to the pupil to share the burden of the same wavefront phase correction, which is different from the dual-DM configuration used for correcting wavefront amplitude and phase [11-13]. The wavefront errors were the inherent aberrations of our breadboard system plus the aberration sample, of which the low-order aberration was basically astigmatism.

\section{B. Wavefront Aberration Partition and Correction}

Given a wavefront slope measurement $\mathbf{S}$ provided by the $\mathrm{SH}$ wavefront sensor, as shown in Fig. 3, the problem of W-T dual-DM wavefront control can

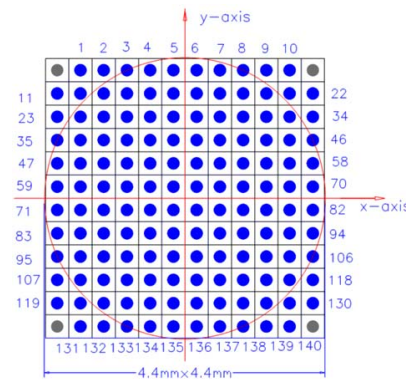

(a)

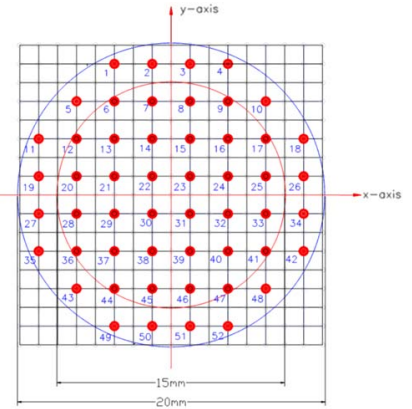

(b)
Fig. 2. (Color online) Actuator distributions of (a) BMC DM and (b) Mirao DM. 
be described as how to determine the actuator vectors $\mathbf{X}$ for the woofer DM and $\mathbf{Y}$ for the tweeter DM from slope data $\mathbf{S}$. The dual-DM AO correction problem is mathematically equivalent to searching for the actuator command vectors $\mathbf{X}$ for the Mirao DM (the woofer) and $\mathbf{Y}$ for the BMC DM (the tweeter) that can minimize the residual wavefront error in a least-squares sense [24],

$$
\psi(\mathbf{X}, \mathbf{Y})=\rho^{2}[\mathbf{A X}+\lambda \mathbf{B Y}, \mathbf{S}]=\|\mathbf{A X}+\lambda \mathbf{B Y}-\mathbf{S}\|_{2}^{2},
$$

where $\mathbf{A}$ and $\mathbf{B}$ are the influence matrices of the Mirao and BMC DMs. The LM $\lambda$ was used to mathematically integrate the two DMs into an "imaginary" monolithic DM, creating a composite influence ma$\operatorname{trix} \mathbf{C}=\left[\begin{array}{ll}\mathbf{A} & \lambda \mathbf{B}\end{array}\right]$ for the "imaginary" DM. The LM method was successfully exemplified in the integration of segment alignment control and segment phase control [32].

By minimizing the objective function $\psi(\mathbf{X}, \mathbf{Y})$, the actuator command vectors $\mathbf{X}$ and $\mathbf{Y}$ can be obtained by $[\underline{24}]$

$$
\left[\begin{array}{l}
\mathbf{X} \\
\mathbf{Y}
\end{array}\right]=\left[\begin{array}{cc}
\mathbf{A}^{T} \mathbf{A}+\beta_{1} \mathbf{I}_{1} & \lambda \mathbf{A}^{T} \mathbf{B} \\
\lambda \mathbf{B}^{T} \mathbf{A} & \lambda^{2} \mathbf{B}^{T} \mathbf{B}+\beta_{2} \mathbf{I}_{2}
\end{array}\right]^{-1}\left[\begin{array}{c}
\mathbf{A}^{T} \\
\lambda \mathbf{B}^{T}
\end{array}\right] \mathbf{S}
$$

where $\beta_{1}$ and $\beta_{2}$ are the damping factors for the Mirao $\mathrm{DM}$ and the BMC DM, respectively, and $\mathbf{I}_{1}$ and $\mathbf{I}_{2}$ are identity matrices. Due to the correlation between the two DMs, the normal matrix $\mathbf{C}^{\mathbf{T}} \mathbf{C}$ is usually badly conditioned. The DLS method is used to suppress the correlation between the two DMs, yielding a wellconditioned normal matrix. The DLS method was first introduced by Levenberg and later on was widely used in optical design [33-39]. In this paper, we explore the LM-based DLS $\overline{\mathrm{AO}}$ control algorithm in wavefront aberration sorting and correction across iterations.

\section{Correlation Problem}

For a given wavefront aberration, the wavefront correlation problem in dual-DM AO controls arises from the uncertainty of the required actuator displacements between the two DMs. In the W-T AO systems, the two DMs are not independent, as they share the same wavefront sensor and contribute to the same wavefront correction via the influence matrices A

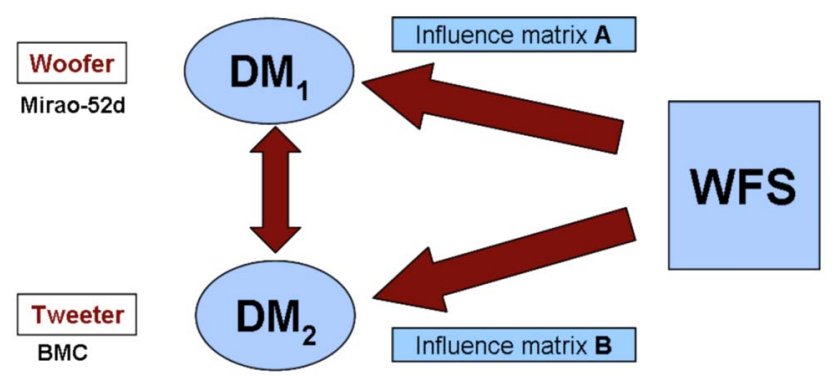

Fig. 3. (Color online) Problem description of W-T dual-DM AO correction. and B. For instance if one DM "pushes" and another DM "pulls" the same region of wavefront, then the total change in wavefront could be minimal while the mirrors were actually using a significant amount of deflections. On the other hand, for a given wavefront correction, one DM can contribute more and the other DM less, or vice versa, while in both scenarios the AO control equation can still be satisfied. For this reason the correlation between two identical DMs is at a minimum only when they are mapped in an interlaced geometry, as shown in Fig. $\underline{4}$. A dual-DM AO system with the illustrated mapping will provide an equivalent correction of a single-DM AO system with the doubled actuator density. However, typically the DMs are not identical or perfectly interleaved; therefore, the inherent correlation between actuators can be serious and the dual-DM AO control is sensitive to sensor noise and liable to DM saturation due to the opposed action of the DMs.

\section{Experimental Results}

\section{A. Wavefront Correction Results}

For the present breadboard AO system, the condition number of a normal matrix was defined as the ratio of maximum to minimum eigenvalues (Fig. 5). It is a measure of numerical stability of the normal matrix. The numerical stability improves if the condition number of the matrix decreases. By damping conditioning (for example, $\beta_{1}=100$ and $\beta_{2}=0.05$ ), the condition number of the composite normal matrix $\mathbf{C}^{\mathbf{T}} \mathbf{C}$ was decreased from $5.38 \times 10^{9}$ to $6.77 \times 10^{6}$, and the correlation between the two DMs suppressed. Note that in Fig. 5, due to different units of the voltages to the Mirao and BMC used in influence matrix calibration, the condition number was still numerically large after damping conditioning. However, it would not be a problem because we can normalize the voltage units to reduce the condition number if the precision of numerical computation is an issue.

Figure 6 shows wavefront correction of the first four iterations of the W-T AO breadboard experimental system. The wavefront root mean square (RMS) error was efficiently reduced from $0.443 \mu \mathrm{m}$ to $0.078 \mu \mathrm{m}$ after its first iteration, then to $0.044 \mu \mathrm{m}$ after its second iteration, and finally to $0.034 \mu \mathrm{m}$

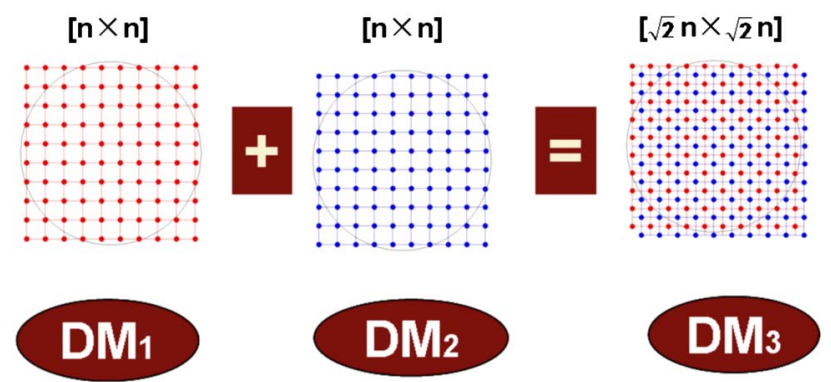

Fig. 4. (Color online) Actuator-interlaced dual-DM AO. It is equivalent to a single-DM AO with double actuator density. 


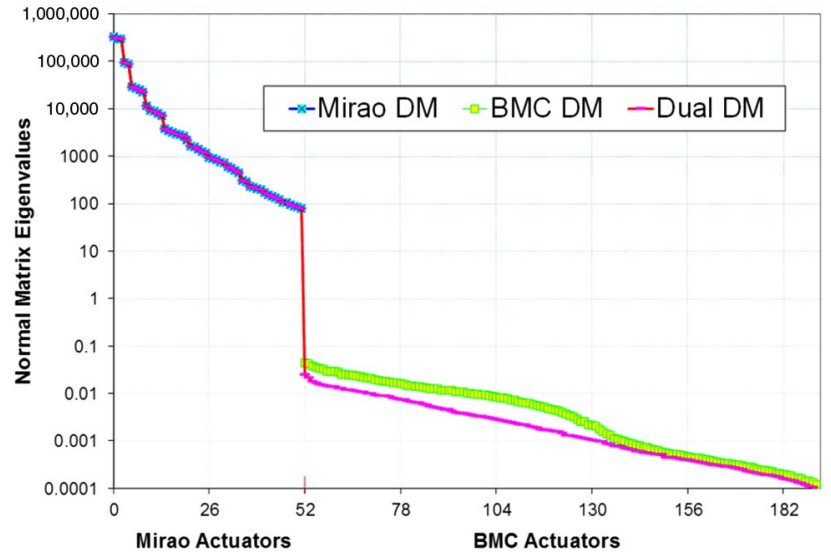

Fig. 5. (Color online) Eigenvalue spectrum of normal matrices $\mathbf{A}^{\mathbf{T}} \mathbf{A}$ (blue curve), $\mathbf{B}^{\mathbf{T}} \mathbf{B}$ (green curve), and $\mathbf{C}^{\mathbf{T}} \mathbf{C}$ (red curve) with LM $\lambda=1$. Mirao actuators were numbered as $0-51$, and the BMC actuators were numbered as 52-191. Due to the different voltage units adopted, the eigenvalues of the Mirao DM are much larger than those of the BMC DM.

after its third iteration, where the wavefront error maps were the measurements from the $\mathrm{SH}$ sensor. Figure 7 shows the improvements of PSF of the dual-DM AO breadboard system in its first five iterations. Figure 7(a) is the PSF before correction, and Figs. 7(b) $7(\mathrm{f})$ are the PSFs in the process of $\mathrm{AO}$ correction, where the Strehl ratio (SR, increased from $4.5 \times 10^{-8}$ to 0.91 ) was computed from wavefront RMS error [40]. We can see that the PSF quality increased as the wavefront RMS error decreased. Figure 8 plots the real-time wavefront residual error of the $\overline{\mathrm{W}}-\mathrm{T}$ LM DLS AO for the first 60 iterations for small amplitude wavefront error $(0.443 \mu \mathrm{m}$ in RMS, as shown in Fig. 6) (Curve 1), and for the large amplitude wavefront error ( $3.5 \mu \mathrm{m}$ in RMS, Curve 2). Because of the nonlinearity of deflection-to-voltage relation of each DM, the correction accuracy for large-amplitude wavefront errors was not as high as that for small amplitude wavefront errors. This can be seen in Curve 3, where we used a dual influence matrix (DIM) AO correction to improve wavefront correction accuracy up to the level of $\mathrm{AO}$ correction obtained for small-amplitude wavefront errors (Curve 1) [25]. Figure 9 shows the wavefront corrections of a single Mirao DM AO for wavefront error of $3.22 \mu \mathrm{m}$ in RMS in comparison to a single BMC DM $\mathrm{AO}$ for wavefront aberration $0.35 \mu \mathrm{m}$ in RMS. For the Mirao DM AO, the DIM AO method was an efficient approach for improving AO correction accuracy (from 0.085 to less than $0.04 \mu \mathrm{m}$ in RMS). Due to its limited dynamic range, the single BMC DM had limited wavefront correction accuracy $(0.13 \mu \mathrm{m}$ RMS in average), even though the BMC DM can offer a better performance than the Mirao DM for the very small amplitude wavefront errors.

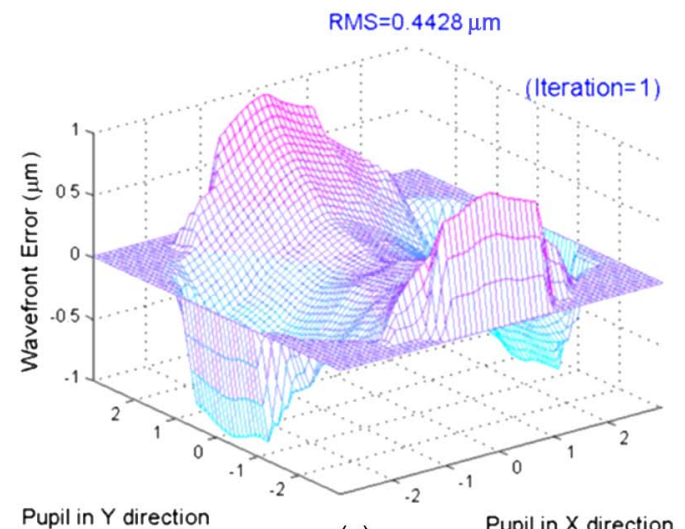

Pupil in $Y$ direction

(a)

Pupil in $\mathrm{X}$ direction RMS $=0.0441 \mu \mathrm{m}$

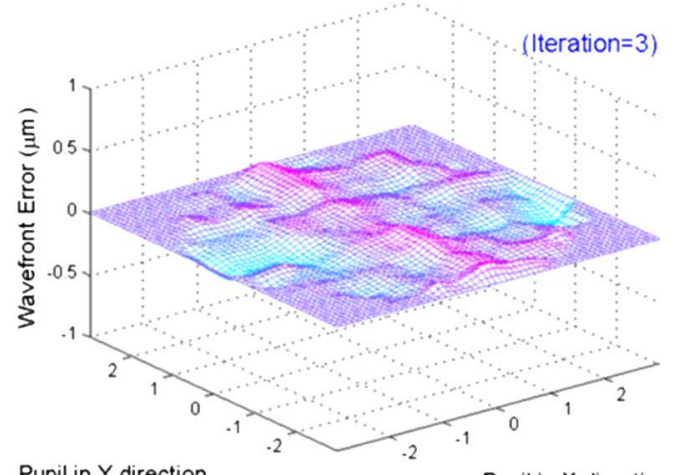

(c)

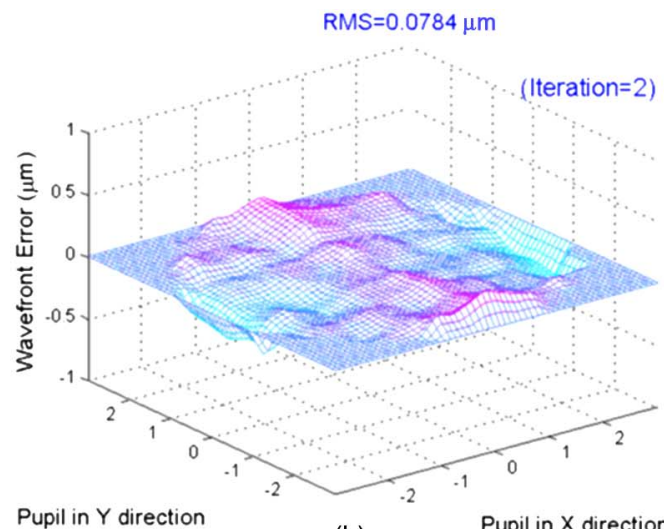

(b)

RMS $=0.0337 \mu \mathrm{m}$

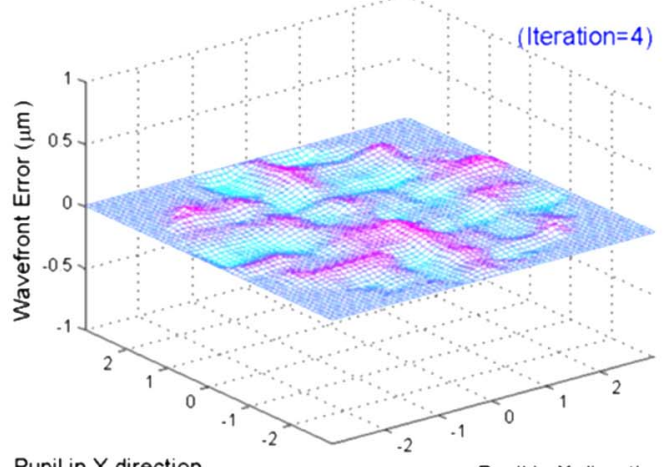

(d)
Pupil in $\mathrm{X}$ direction

Fig. 6. (Color online) Wavefront error reduction across four iterations of dual-DM AO correction with the damping factors $\beta_{1}=100$ and $\beta_{1}=0.05$, LM $\lambda=1.0$, and control gain of 0.75 . 


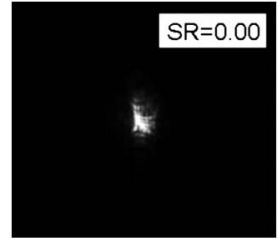

(a)

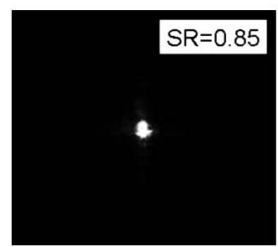

(d)

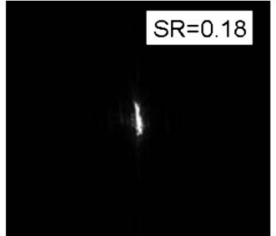

(b)

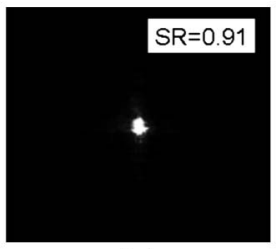

(e)

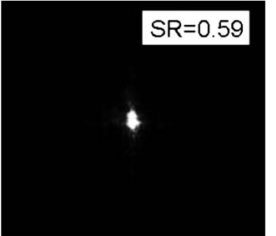

(c)

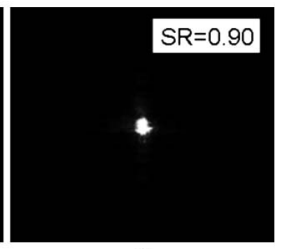

(f)
Fig. 7. PSF of the breadboard system across dual-DM AO iterations. (a) PSF before AO correction. (b)-(f) PSFs during AO correction at first, second, third, fourth, and fifth iterations. The Strehl ratio for each PSF was estimated from the real-time wavefront RMS error.

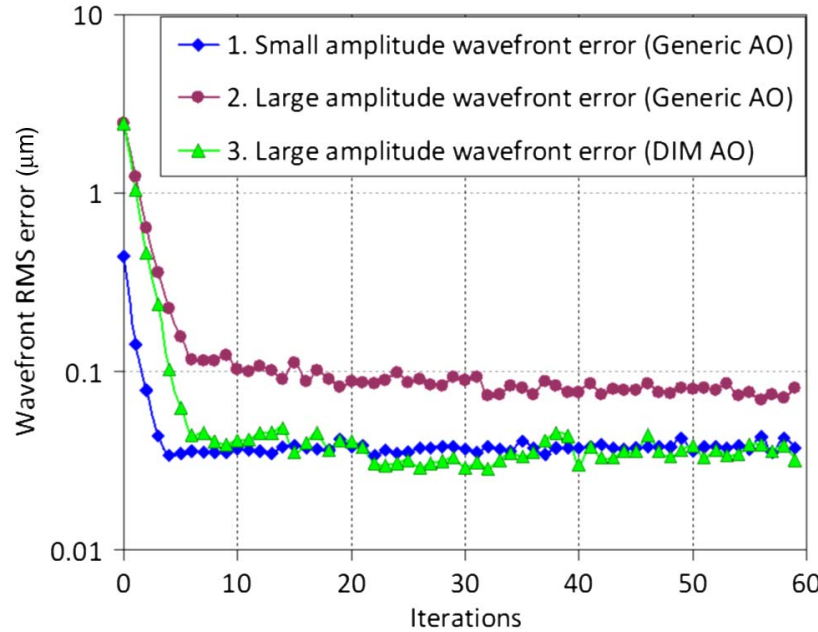

Fig. 8. (Color online) Wavefront RMS error reduction of dual-DM $\mathrm{AO}$ (generic AO) for small wavefront aberrations (Curve 1) and comparison of the generic AO and DIM AO for large amplitude wavefront aberrations (Curves 2 and 3). Curve 1 and Fig. $\underline{6}$ use the same data.

\section{B. Optimizations of AO Control Parameters}

To evaluate the control performance for a range of damping factors, the final wavefront residual RMS error was used as the metric. The damping factors for the Mirao DM were varied from 0.01 to 10,000 , and those for the BMC DM were varied from 0.001 to 1000 . The resulting grid search optimization of the two damping factors is shown in Fig. 10. When we adopted the damping factors approximately between 500 and 2000 for the Mirao DM and between 0.004 and 0.01 for the BMC DM, the final wavefront residual RMS error would be reduced to less than $0.03 \mu \mathrm{m}$, and the condition number would be improved by up to four orders of magnitude. Apparently, the best damping factors for the Mirao and BMC DMs for the breadboard system were approximately 1000 and 0.005 , respectively.

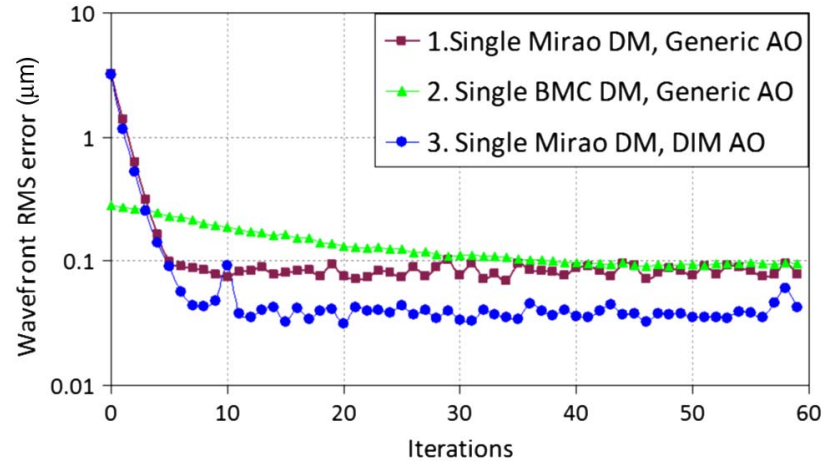

Fig. 9. (Color online) Wavefront RMS error reduction of single $\mathrm{BMC} \mathrm{DM} \mathrm{AO}$ (generic AO) for small wavefront aberrations and comparison of the generic AO and DIM AO with single Mirao DM for large-amplitude wavefront aberrations.

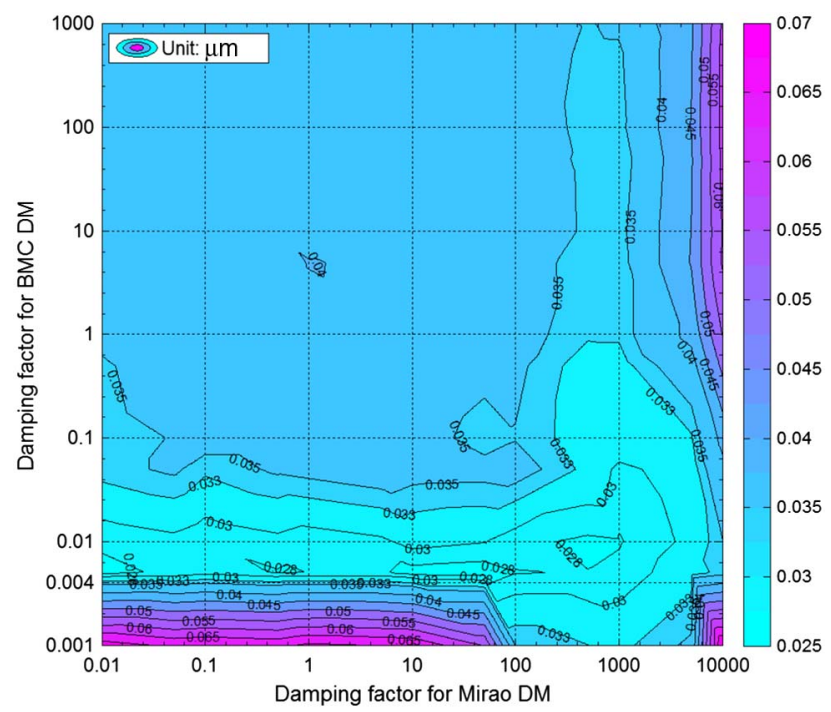

Fig. 10. (Color online) Damping factor optimization for W-T dual$\mathrm{DM}$ AO correction. This figure shows the contour plot of the final wavefront residual RMS error as the function of the damping factors. We can see that the optimum values of the two damping factors are at 1000 for the woofer DM and 0.004 for the tweeter DM, respectively.

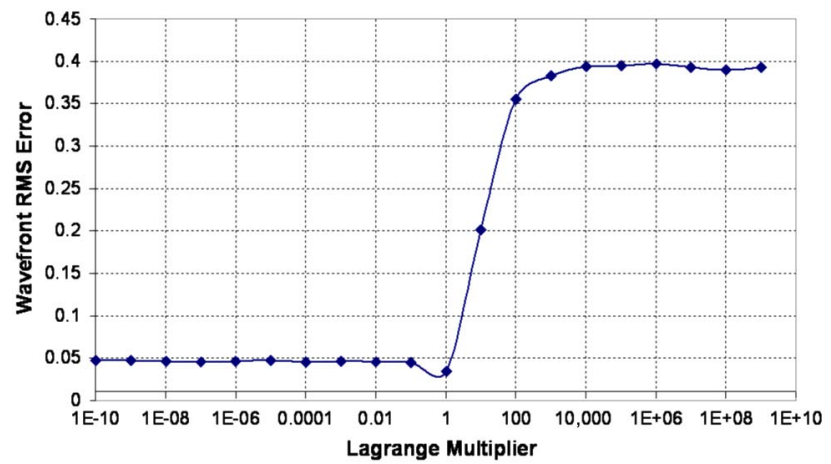

Fig. 11. (Color online) Optimization of LM for the LM DLS algorithm. 


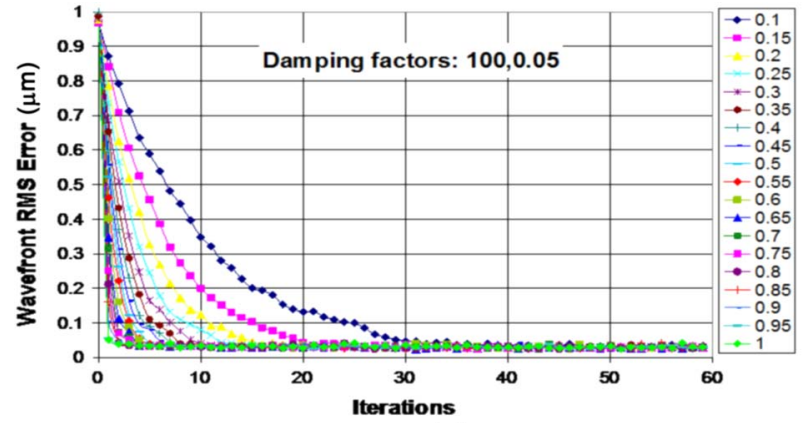

(a)

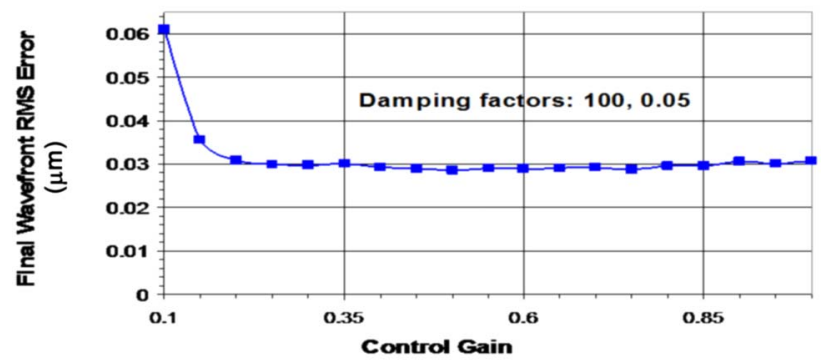

(b)

Fig. 12. (Color online) (a) Optimization of control gain. (b) Final averaged wavefront RMS error (16-60 iterations) as a function of control gain.

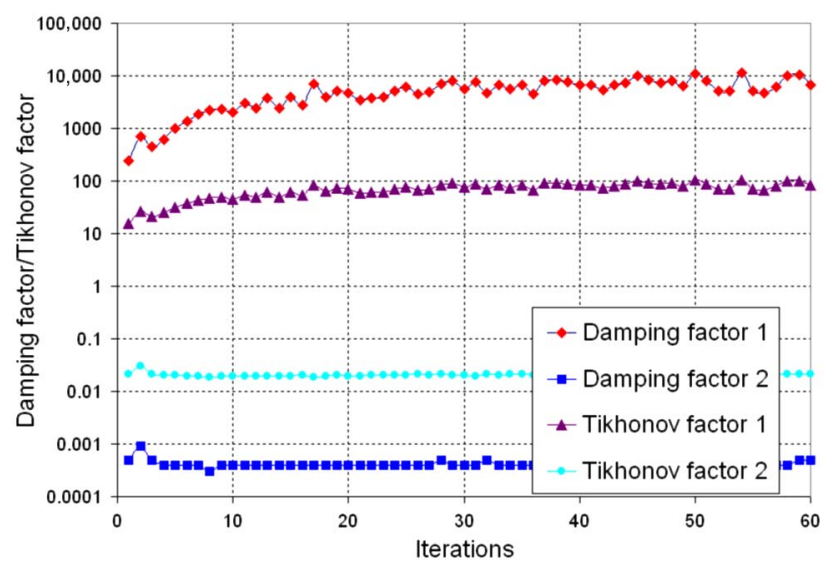

Fig. 13. (Color online) Bayesian estimation of the damping factors. The damping factor is square of the Tikhonov factor, where the damping factor $1\left(\beta_{1}\right)$ is for the Mirao DM, and damping factor $2\left(\beta_{2}\right)$ is for the BMC DM.

The role of LM is to integrate two separate influence matrices into a single influence matrix, and its value is usually set to 1 but can be varied. To examine the impact of the LM on AO correction performance, we varied the LM from $10^{-10}$ to $10^{10}$ and again used the wavefront residual RMS error as the optimization metric. The optimization was



(a)

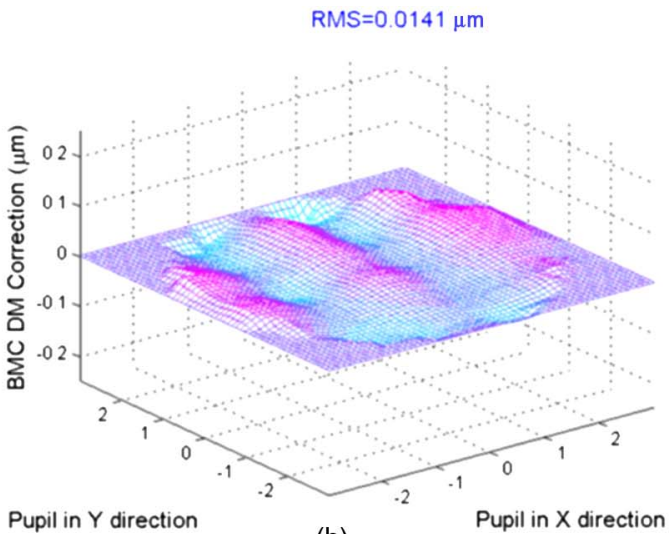

(b)

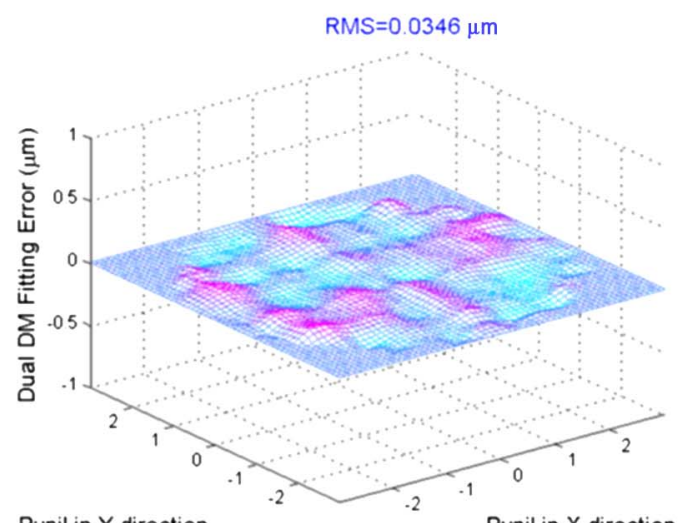

(c)

Fig. 14. (Color online) Simulation of LM DLS dual-DM AO correction (1-Step) for the given wavefront shown in Fig. 6(a) (control gain set to 1), where (a), (b) are the expected wavefront corrections by the Mirao DM and the BMC DM, respectively, and (c) is the expected wavefront fitting error of the dual-DM AO. 


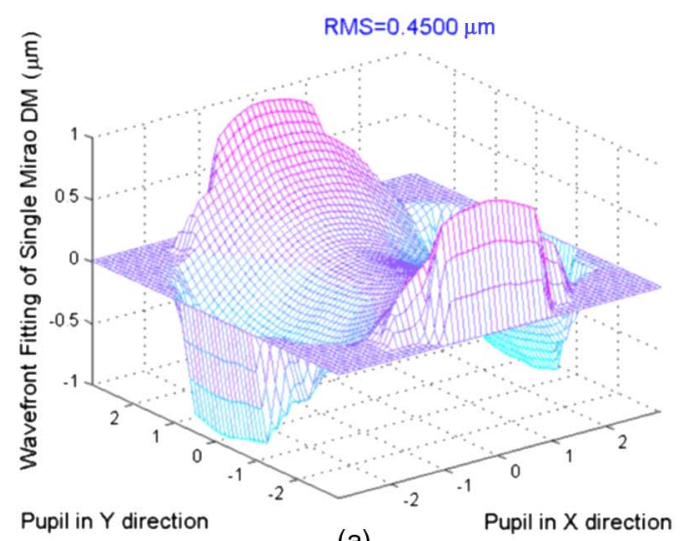

(a)

RMS $=0.4039 \mu \mathrm{m}$

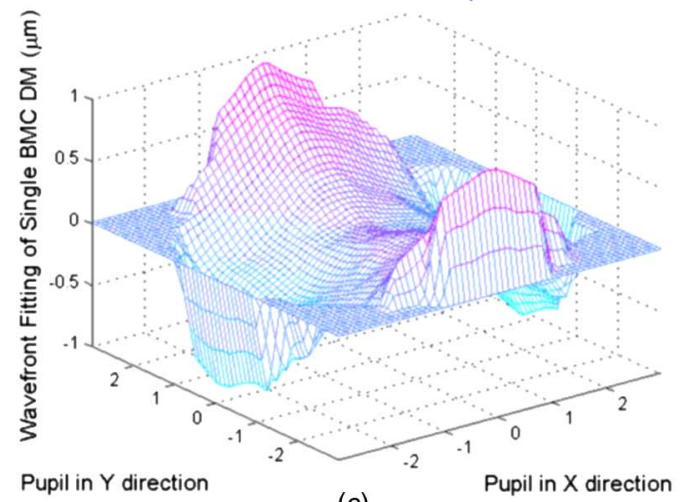

(c)

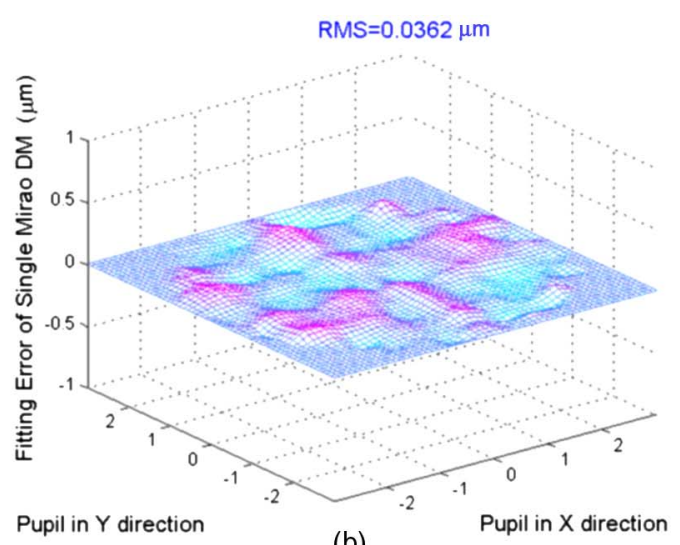

(b)

RMS $=0.0604 \mu \mathrm{m}$

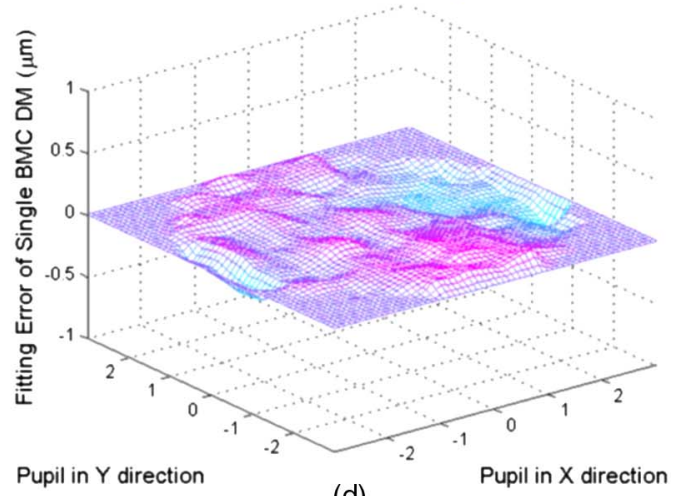

(d)

Fig. 15. (Color online) Simulations of single DM AO correction, where (a), (c) are the expected wavefront corrections by single Mirao DM and single BMC DM, respectively, and (b), (d) are their corresponding wavefront fitting errors.

performed with the damping factors of $\beta_{1}=500$ and $\beta_{2}=0.05$, and a control gain of 0.6 , and the result is shown in Fig. 11, in which the wavefront aberrations were generated by trial lenses of -0.5 diopter (D) astigmatism plus 0.12D sphere.

Closely related to the damping factors adopted is the control gain. It should be determined by the sampling time of the system, the temporal response of the DMs, and the noise level. Usually a value of 0.4 is the limit for a system with a one-frame measurement delay; however, in our system, the damping factor complicates the calculation a bit, and so we determined it experimentally. Figure 12(a) shows the wavefront RMS error reduction curves of a static aberration correction for different control gains, and Fig. 12(b) is the final wavefront RMS error optimized with the control gain.

\section{Discussion}

\section{A. Control Parameter Selections}

The role of the damping factor is for suppressing the correlation between mirrors, controlling the error propagation, and adjusting the correction amplitude in each step. Too small a damping factor will generate little effect on the actuators with large eigenvalues, while too large a damping factor will damp down the small-eigenvalue actuators to zero. Relating Fig. 10 with Fig. 5 , we can confirm that the opti- mum damping factor for a DM is approximately located at the median of the eigenvalue spectrum, which is reasonable because the median represents the equilibrium point for error damping among all actuators. For the breadboard system, the eigenvalue median was about 1000 for the Mirao DM and 0.004 for the BMC DM. Similar to our observation, Matsui and Tanaka also suggested using the median of eigenvalues of the normal Jacobian matrix as the appropriate damping factor for nonlinear LS optimization in optical design [39].

Another approach to optimizing the damping factor is based on Bayes' theorem. Based on the stable equilibrium of $\mathrm{AO}$ correction, we can assume that DM commands $X$ and $Y$ are statistically random variables of multivariate normal distribution with zero mean and standard deviations of $\sigma_{X}$ and $\sigma_{Y}$, and the slope errors $S$ are independent random variables with zero mean and standard deviation $\sigma_{S}$. Under this assumption, the Tikhonov-regularized solution is the most probable solution according to Bayes' theorem. We can obtain the Tikhonov factors $\alpha_{1}=\sigma_{S} / \sigma_{X}$ for the Mirao DM and $\alpha_{2}=\sigma_{S} / \sigma_{Y}$ for the BMC DM [41]. Then the damping factors for the two DMs are $\beta_{1}=\left(\sigma_{S} / \sigma_{X}\right)^{2}$ and $\beta_{2}=\left(\sigma_{S} / \sigma_{Y}\right)^{2}$. Applying the real slope data from our breadboard system, we estimated the damping factors for the Mirao $\mathrm{DM}$ as 5365 and for the BMC DM as 0.0004 (Fig. 13). Obviously, such a solution is not optimal according to 
Fig. 10, but it is a working solution for the woofer and tweeter DMs that are not truly independent.

The optimum value for the LM was 1 (Fig. 11). When the LM was smaller than 1, the residual wavefront RMS increased from 0.03 to $0.05 \mu \mathrm{m}$, because in this case the AO correction was dominated by the Mirao correction, and the residual wavefront RMS error was actually the accuracy of single Mirao DM correction. When the LM was larger than 1, the BMC correction began to dominate, and the residual RMS error was increased to $0.4 \mu \mathrm{m}$, because with the large LM $(\lambda>100)$ all the wavefront error was applied to the BMC DM for correction, and it saturated.

Generally in AO control, a gain higher than 0.6 is usually avoided, because the system can go into oscillations from very small disturbances. However, the gain interacts with the control damping in our algorithm. Higher damping can allow higher gain, although of course this does not necessarily speed up convergence of correction, but it can provide a better conditioned control matrix and therefore a more stable control. Usually the gain will be smaller for a system with dynamic aberrations (such as the eye). As shown in Fig. 12(b), the best working gain lies between 0.2 and $0.8 \mu \mathrm{m}$ for this breadboard $\mathrm{AO}$ system.

\section{B. Wavefront Fitting Computation: "1-Step" versus "2-Step"}

To establish a metric for evaluating wavefront correction with an algorithm, we defined the wavefront fitting error as the expected wavefront residual error obtained by subtracting the theoretical fitting wavefront of the DM (or DMs) from a given wavefront. Given the aberrated wavefront of Fig. 6(a), Figs. 14(a) and $14(\mathrm{~b})$ are the theoretical fitting wavefronts by the woofer DM and the tweeter DM. They are the expected wavefront corrections that the two DMs can provide with the LM DLS algorithm [Eq. (2)], and Fig. 14(c) is the wavefront fitting error of the dual DMs. Using the same wavefront of Fig. 6(a) and with the traditional singular value decomposition method, Figs. 15(a) and 15(c) are the theoretical fitting wavefronts by the single Mirao DM and by the single BMC DM, respectively, and Figs. 15(b) and 15(d) are their corresponding wavefront fitting errors.

Instead of the single-step (1-Step) inverse computation defined by Eq. (2), the dual-DM correction can be computed in a two-step process (2-Step): given a wavefront aberration, the woofer DM command can be first computed by treating it as a single-DM correction, and then we numerically subtract the woofer correction from the original wavefront and

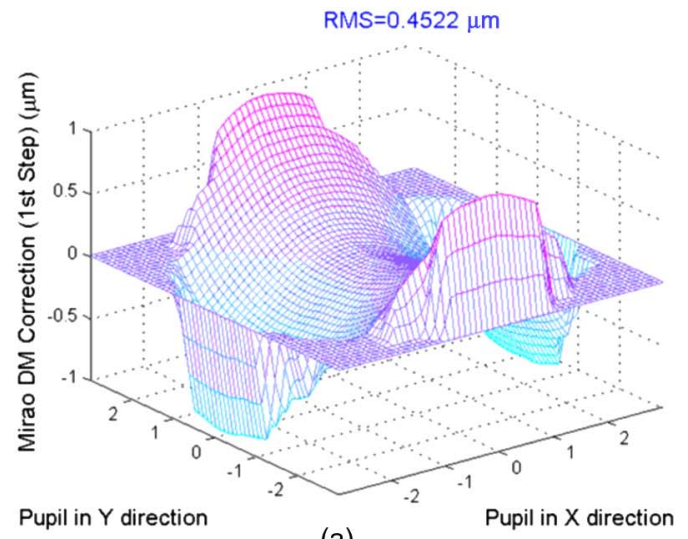

(a)

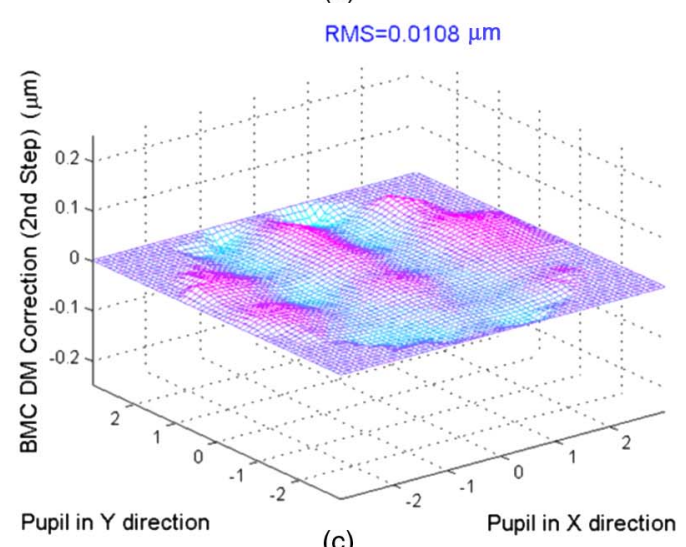

(c)

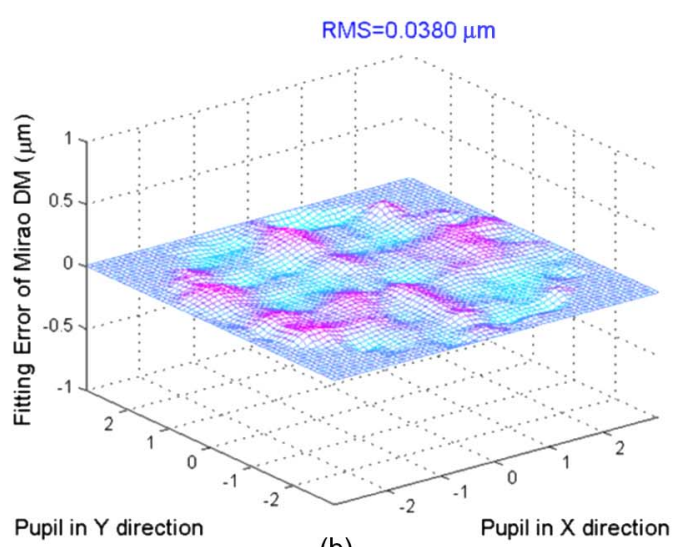

(b)

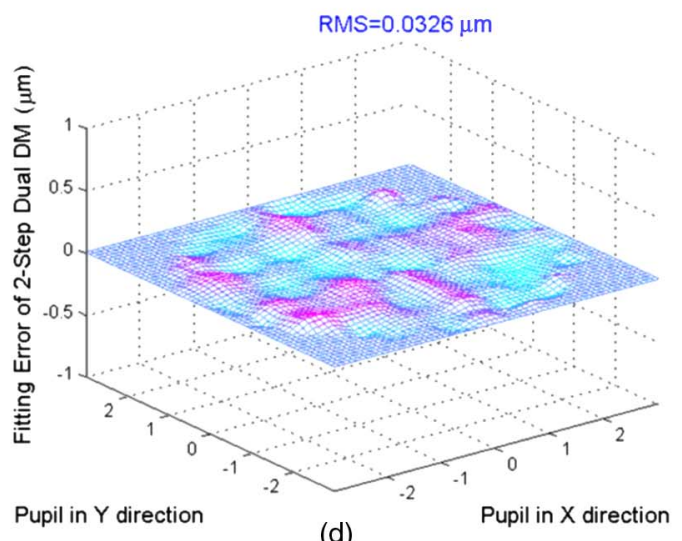

(d)

Fig. 16. (Color online) Simulation of LM DLS AO correction with the 2-Step computation for the given wavefront shown in Fig. 6(a) (control gain set to 1), where (a) is the expected wavefront correction by single Mirao DM $\left(\beta_{1}=100\right)$, (b) is the corresponding wavefront fitting error of Mirao DM, (c) is the expected wavefront correction by the $\operatorname{BMC~DM}\left(\beta_{2}=0.05\right)$ for the wavefront fitting error of (b), and (d) is the final wavefront fitting error of the 2-Step computation. 


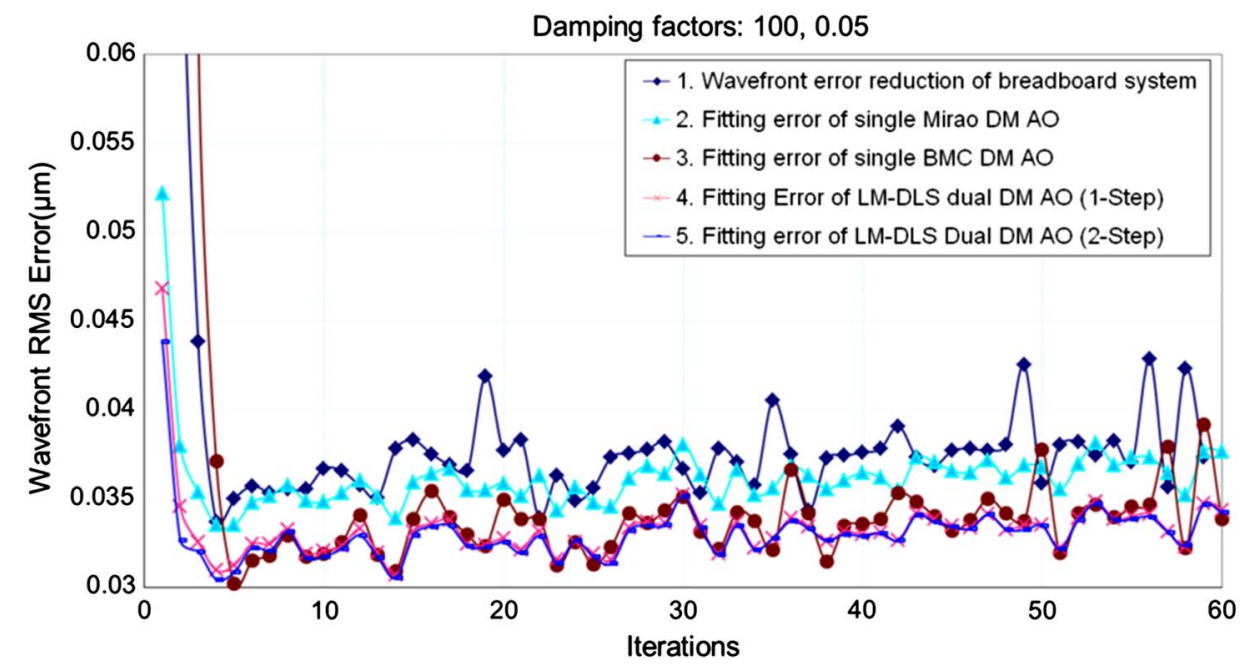

Fig. 17. (Color online) Comparison of wavefront fitting errors of single Mirao DM (Curve 2), single BMC DM (Curve 3), LM DLS dual-DM AO (1-Step) (Curve 4), and (2-Step) (Curve 5). Wavefront RMS error reduction of the dual-DM AO (Curve 1), which used the same data as in Fig. $\underline{8}$, provided a reference for the wavefront fitting errors.

obtain the residual wavefront (i.e., wavefront fitting error of woofer DM), from which the tweeter DM actuator commands can be computed in the second step. If there is no correlation between the two DMs, then the 1-Step and the 2-Step inverse computations are equivalent. Given that the woofer DM has much larger dynamic range than the tweeter DM, it is more secure to use the woofer DM as the first fitting DM in the 2-Step computation even if the two DMs have correlations. The tweeter DM can fit the residual wavefront error of the first step, and the two fitting optimization processes are independent. The W-T dual-DM wavefront fitting with the 2-Step computation is shown in Fig. 16. Given the original wavefront error shown in Fig. 6(a), Fig. 16(a) is the estimated wavefront correction by single Mirao DM; Fig. 16(b) is the corresponding wavefront fitting error. Figure 16(c) is the estimated wavefront correction by the $\overline{\mathrm{BMC}} \mathrm{DM}$ for the wavefront residual error in Fig. 16(b), and Fig. 16(d) is the final wavefront fitting error of the 2-Step computation. Comparing the fitting errors of 1-Step and 2-Step methods in Fig. 14(c) and Fig. 16(d), we can see that the two approaches are essentially equivalent $(2 \mathrm{~nm}$ RMS difference).

Figure 17 plots the above-mentioned wavefront fitting errors (1-Step and 2-Step) for the first 60 iterations (Curves 2-5) in comparison with the real-time wavefront residual error of the W-T LM DLS AO (Curve 1), where Curves 1 in Fig. 17 and Fig. 8 represent the same data. We can see that the 1-Step and 2-Step wavefront fitting errors of LM DLS AO are actually superposed after the AO converged, confirming that the two computations were indeed equivalent for the breadboard system. The real-time wavefront residual error is always larger than the wavefront fitting errors (for both 1-Step and 2-Step approaches). The wavefront fitting error of single $\mathrm{BMC} \mathrm{DM}(0.034 \mu \mathrm{m}$ in average $)$ is smaller than that of single Mirao DM $(0.036 \mu \mathrm{m}$ in average $)$, and the wavefront fitting error of the LM DLS dual-DM correction (Curve 4 or Curve 5, both $0.033 \mu \mathrm{m}$ in average) is smaller than that of any single-DM correction. Of course, when the low-order aberrations dominate the wavefront error, the performance of single Mirao $\mathrm{AO}$ is very close to that of dual-DM AO. However, simulation showed that, for a population of 100 eyes using the Thibos model, the static ocular aberrations correction with the Mirao DM (with focus and astigmatism removed) can reach a mean RMS wavefront error of less than $0.03 \mu \mathrm{m}[14,42, \underline{43}]$.

To calibrate the wavefront sensing accuracy, the $\mathrm{SH}$ grid was optimized to its best condition, and a $0.014 \mu \mathrm{m}$ wavefront RMS error due to SH centroiding error was measured. As shown in Fig. 17, on average the wavefront fitting error of the LM-DLS dual-DM AO (Curve 4) has a deviation of $0.017 \mu \mathrm{m}$ in RMS from the real wavefront residual error (Curve 1), which implies that the average wavefront residual RMS error $0.037 \mu \mathrm{m}$ (Curve 1) included the wavefront fitting RMS error $0.033 \mu \mathrm{m}$ (Curve 4), SH sensing RMS error $0.014 \mu \mathrm{m}$, and actuator positioning RMS error $0.01 \mu \mathrm{m}$.

\section{Conclusion}

We developed a breadboard system for the W-T dualDM AO, validated the LM-based zonal DLS control algorithm on this system, and explored the AO performance over a wide range of parameters. We confirmed that the zonal DLS control algorithm can complementarily partition the wavefront aberrations for the woofer and tweeter DMs according to their individual influence functions just as if the actuators of the two DMs belong to a single DM. In our measurements with the static aberration the wavefront control accuracy of LM DLS algorithm was much higher than a single DM. This may not be surprising because there are more actuators and the W-T AO has a more diverse set of influence functions; however, it confirms that the W-T approach can use two 
less-expensive mirrors to provide a high-accuracy, high-stroke composite mirror. In practice we have found that we can use a low-stroke MEMS mirror in conjunction with the Mirao DM to achieve a high-accuracy W-T AO, and it suggests that it might be possible to use even lower actuator count (and hence lower cost) electromagnetic mirrors as the woofer, too. With the LM-based DLS algorithm, we can integrate multiple stroke-limited DMs to obtain an "imaginary" DM with extraordinary stroke capability and ultrahigh spatial resolution for biomedical AO imaging and for astronomy observation [44].

After the LM-based DLS algorithm was proposed and validated, we implemented it in the wide field $\mathrm{W}$ T dual-DM scanning laser ophthalmoscope (AOSLO) system for in vivo retinal imaging for human subjects, and it has been shown to be very efficient [26]. The W-T AOSLO system can provide the stable realtime retinal imaging that is very close to diffraction limit. In our AOSLO system, the global tip/tilt of wavefront in the slope data is filtered out, therefore the tip/tilt of the wavefront was not corrected, yet the LM-based DLS algorithm has the capability to correct wavefront tip/tilt. In the algorithm implementation, the boundary condition of the control pupil should be taken care carefully so that the improvement in wavefront control accuracy gained from the efficient algorithm will not be flooded by the loworder wavefront error induced from boundary slope noise [45].

The LM DLS algorithm with either 1-Step or 2Step computation provides similar wavefront fitting errors, which implied that the correlation between the woofer and tweeter was not significant. The 1Step LM DLS was approved to be efficient for in vivo retinal imaging because the woofer and tweeter DMs can work simultaneously and complementarily [26]. The 2-Step approach is ideal in that the woofer and the tweeter DMs contribute independently to the simultaneous corrections. For our breadboard system with optimized control parameters, the wavefront can be controlled to be less than $0.03 \mu \mathrm{m}$ in RMS for small aberrations; however, to obtain a diffractionlimited PSF (Strehl ratio of 0.92), we need to better calibrate the $\mathrm{SH}$ wavefront sensor.

This work was supported by NIH EY04395 and NIH EY14375.

\section{References}

1. A. Guirao and P. Artal, "Off-axis monochromatic aberrations estimated from double pass measurements in the human eye," Vis. Res. 39, 207-217 (1999).

2. D. A. Atchison, "Higher order aberrations across the horizontal visual field," J. Biomed. Opt. 11, 34026 (2006).

3. D. A. Atchison, N. Pritchard, and K. L. Schmid, "Peripheral refraction along the horizontal and vertical visual fields in myopia," Vision Res. 46, 1450-1458 (2006).

4. A. Mathur, D. A. Atchison, and D. H. Scott, "Ocular aberrations in the peripheral visual field," Opt. Lett. 33, 863-865 (2008).

5. X. Wei and L. Thibos, "Modeling the eye's optical system by ocular wavefront tomography," Opt. Express 16, 20490-20502 (2008).
6. L. Lundström, A. Mira-Agudelo, and P. Artal, "Peripheral optical errors and their change with accommodation differ between emmetropic and myopic eyes," J. Vis. 9 (6), 17 (2009).

7. W. N. Charman and G. Heron, "Fluctuations in accommodation: a review," Ophthalm. Physiol. Opt. 8, 153-163 (1988).

8. D. C. Chen, S. M. Jones, D. A. Silva, and S. S. Olivier, "Highresolution adaptive optics scanning laser ophthalmoscope with dual deformable mirrors," J. Opt. Soc. Am. A 24, 1305-1312 (2007).

9. R. J. Zawadzki, S. S. Choi, S. M. Jones, S. S. Oliver, and J. S. Werner, "Adaptive optics-optical coherence tomography: optimizing visualization of microscopic retinal structures in three dimensions," J. Opt. Soc. Am. A 24, 1373-1383 (2007).

10. B. Cense, E. Koperda, J. M. Brown, O. P. Kocaoglu, W. Gao, R. S. Jonnal, and D. T. Miller, "Volumetric retinal imaging with ultrahigh-resolution spectral-domain optical coherence tomography and adaptive optics using two broadband light sources," Opt. Express 17, 4095-4111 (2009).

11. M. C. Roggemann and D. J. Lee, "Two-deformable-mirror concept for correcting scintillation effects in laser beam projection through the turbulent atmosphere," Appl. Opt. 37, 4577-4585 (1998).

12. J. D. Barchers, "Application of the parallel generalized projection algorithm to the control of two finite-resolution deformable mirrors for scintillation compensation," J. Opt. Soc. Am. A 19, 54-63 (2002).

13. J. D. Barchers, "Closed-loop stable control of two deformable mirrors for compensation of amplitude and phase fluctuations," J. Opt. Soc. Am. A 19, 926-945 (2002).

14. L. N. Thibos, X. Hong, A. Bradley, and X. Cheng, "Statistical variation of aberration structure and image quality in a normal population of healthy eyes," J. Opt. Soc. Am. A 19 , 2329-2348 (2002).

15. J. Porter, A. Guirao, I. G. Cox, and D. R. Williams, "Monochromatic aberrations of the human eye in a large population," J. Opt. Soc. Am. A 18, 1793-1803 (2001).

16. T. J. Brennan and T. A. Rhoadarmer, "Performance of a woofertweeter deformable mirror control architecture for highbandwidth, high-spatial resolution adaptive optics," Proc. SPIE 6306, 63060B (2006).

17. O. Keskin, P. Hampton, R. Conan, C. Bradley, A. Hilton, and C. Blain, "Woofer-tweeter adaptive optics test bench," in First NASA / ESA Conference on Adaptive Hardware and Systems (IEEE, 2006), pp. 74-80.

18. R. Conan, "Mean-square residual error of a wavefront after propagation through atmospheric turbulence and after correction with Zernike polynomials,” J. Opt. Soc. Am. A 25, 526-536 (2008).

19. K. Morzinski, B. Macintosh, D. Gavel, and D. Dillon, "Stroke saturation on a MEMS deformable mirror for woofertweeter adaptive optics," Opt. Express 17, 5829-5844 (2009).

20. S. Hu, B. Xu, X. Zhang, J. Hou, J. Wu, and W. Jiang, "Doubledeformable-mirror adaptive optics system for phase compensation," Appl. Opt. 45, 2638-2642 (2006).

21. J.-F. Lavigne and J.-P. Véran, "Woofer-tweeter control in an adaptive optics system using a Fourier reconstructor," J. Opt. Soc. Am. A 25, 2271-2279 (2008).

22. R. Conan, C. Bradley, P. Hampton, O. Keskin, A. Hilton, and C. Blain, "Distributed modal command for a two-deformablemirror adaptive optics system," Appl. Opt. 46, 4329-4340 (2007).

23. C. Li, N. Sredar, K. M. Ivers, H. Queener, and J. Porter, "A correction algorithm to simultaneously control dual deformable mirrors in a woofer-tweeter adaptive optics system," Opt. Express 18, 16671-16684 (2010).

24. W. Zou, X. Qi, and S. A. Burns, "Wavefront-aberration sorting and correction for a dual-deformable-mirror adaptive-optics system," Opt. Lett. 33, 2602-2604 (2008).

25. W. Zou and S. A. Burns, "High-accuracy wavefront control for retinal imaging with adaptive-influence-matrix adaptive optics," Opt. Express 17, 20167-20177 (2009).

26. W. Zou, X. Qi, and S. A. Burns, "Woofer-tweeter adaptive optics scanning laser ophthalmoscopic imaging based on 
Lagrange-multiplier damped least-squares algorithm," Biomed. Opt. Express 2, 1986-2004 (2011).

27. http://www.imagine-eyes.com/content/view/45/103/.

28. Specifications of product Model no. $\mu \mathrm{DM} 140-450-\mathrm{E}-\mathrm{AgMgF}$, SN: 09w200\#108-450D16-9, Boston MicroMachines Corporation (2007).

29. Device no. SLD-261-HP1-DIL-SM-PD, http://www .superlumdiodes.com/.

30. Product no. 0300-7.6-S, http://www.st.northropgrumman.com/ aoa/beamcontrol/microoptics/catalog_arrays.html.

31. Product no. UP-1830CL-12B, http://www.uniqvision.com/ $\mathrm{html} / \mathrm{manuals}$.

32. W. Zou, "New phasing algorithm for large segmented telescope mirrors," Opt. Eng. 41, 2338-2344 (2002).

33. K. Levenberg, "A method for the solution of certain nonlinear problems in least squares," Q. Appl. Math. 2, 164-168 (1944).

34. J. Meiron, "Damped least-squares method for automatic lens design," J. Opt. Soc. Am. 55, 1105-1107 (1965).

35. E. Glatzel and R. Wilson, "Adaptive automatic correction in optical design," Appl. Opt. 7, 265-276 (1968).

36. D. R. Buchele, "Damping factor for the least-squares method of optical design," Appl. Opt. 7, 2433-2435 (1968).

37. D. Q. Su and Y. N. Wang, "Automatic correction of aberration in astro-optical system," Acta Astron. Sin. 15(1), 51-60 (1974).
38. D. Q. Su, "Spot-diagram merit function and damped leastsquares method," Opt. Instrum. Technol. 31, 1-12 (1980).

39. H. Matsui and K. Tanaka, "Determination method of an initial damping factor in the damped-least-squares problem," Appl. Opt. 33, 2411-2418 (1994).

40. V. N. Mahajan, "Strehl ratio for primary aberrations in terms of their aberration variance," J. Opt. Soc. Am. 73, 860-861 (1983).

41. http://en.wikipedia.org/wiki/Tikhonov_regularization.

42. N. Devaney, E. Dalimier, T. Farrell, D. Coburn, R. Mackey, D. Mackey, F. Laurent, E. Daly, and C. Dainty, "Correction of ocular and atmospheric wavefronts: a comparison of the performance of various deformable mirrors," Appl. Opt. 47, 6550-6562 (2008).

43. N. Devaney, D. Coburn, C. Coleman, C. Dainty, E. Dalimier, T. Farrell, D. Mackey, and R. Mackey, "Characterisation of MEMs mirrors for use in atmospheric and ocular wavefront correction," Proc. SPIE 6888, 688802 (2008).

44. T. Farrell, "Woofer-tweeter adaptive optics for astronomy," Ph.D. dissertation (National University of Ireland Galway, 2010).

45. W. Zou, X. Qi, G. Huang, and S. A. Burns, "Improving wavefront boundary condition for in vivo high resolution adaptive optics ophthalmic imaging," Biomed. Opt. Express 2, 3309-3320 (2011). 